\title{
El interrogante de lo vivo y sus respuestas ahistóricas: desarrollo histórico del concepto de lo vivo y algunas implicaciones didácticas.
}

Por Correa Mónica ${ }^{1}$

\author{
Recibido: 19-12-2008 \\ Aceptado: 20-04-2009 \\ Porque suele olvidarse con demasiada facilidad \\ Que el brillante espectáculo que ofrece la ciencia actual y, \\ En particular, la ciencia actual de la vida, \\ No es sino la superficie de un proceso histórico \\ Que se remonta a dos mil años atrás o quizá más. \\ Smith: El problema de la vida
}

Resumen. El presente trabajo muestra el desarrollo histórico y epistemológico del concepto de lo vivo, en términos de las diferentes corrientes de pensamiento que han guiado su estudio, como son: El animismo, el mecanicismo, el vitalismo, el organicismo, el pensamiento sistémico y el neomecanicismo. Además, relacionando lo anterior con la formación docente y buscando una reflexión acerca de la importancia de vincular la historia y la epistemología en la enseñanza de las ciencias, se hace una revisión de algunas implicaciones didácticas del abordaje ahistórico de de este concepto en la escuela.

Palabras clave. Lo vivo, historia y epistemología y enseñanza de las ciencias.

Abstract. This work shows the historical and epistemological development of the living concept, from different thinking groups that have guided its study such as: the animism, the mechanics, the vitalism, the organization, the systemic thinking and the neomechanics. More over, it has a relation with the teaching formation and with a reflection about the importance of including the history and the epistemology in the science's teaching, besides it makes a review of the didactical implications of the ahistorical taking of this concept at school.

Key words. The living, history and epistemology, and science's teaching.

En el presente escrito se presentan los avances, obstáculos, problemas y demás acontecimientos que permitieron el desarrollo histórico y epistemológico del concepto de lo vivo, en términos de las diferentes corrientes de pensamiento que han guiado su estudio. Dada la importancia que en los procesos de formación de docentes se asigna a la relación entre historia y epistemología de las ciencias y enseñanza de las ciencias, se hace una reflexión sobre algunas implicaciones didácticas que tiene el abordaje ahistórico de este concepto en la escuela. Lo anterior

${ }^{1}$ Estudiante Departamento de Biología. Universidad Pedagógica Nacional.

dbi341 mcorrea@pedagogica.edu.co 
se desarrolla a partir de tres apartados: El interrogante de lo vivo y sus respuestas ahistóricas, desarrollo histórico del concepto de lo vivo y algunas implicaciones didácticas del tratamiento ahistórico del concepto de lo vivo.

\section{EL INTERROGANTE DE LO VIVO Y SUS RESPUESTAS AHISTÓRICAS.}

¿Qué es la vida? hace más de medio siglo, el físico austriaco Edwin Schrödinguer (1984), escribió un libro titulado de esta manera. Sin embargo, incluso antes de esa fecha diversos personajes en diferentes momentos históricos han intentado encontrarle respuesta, respuestas que van desde posturas teológicas, filosóficas, físicas, hasta respuestas que argumentan que responder esta pregunta no es útil ni fácil, ya que todos sabemos lo que es la vida, desde el vivir humano, pero no podemos explicarlo (Varela, 2002)

Son tantos los esfuerzos realizados por responder esta pregunta, que en el siglo XIX surgió una ciencia cuyo objeto de estudio gira en torno a este interrogante; como diría Smith (1975) en su libro el problema de la vida "La historia de la conquista de una respuesta a esta pregunta constituye en gran medida la historia del crecimiento de la ciencia de la Biología". Además es posible mostrar que la conquista de esta respuesta está en relación con la transformación de la pregunta que interroga por la vida, ya que aunque "vida y vivo" parezcan términos sinónimos, la vida es una noción que no permite dar cuenta del fenómeno vivo, por cuanto es sólo el resultado de una evidencia y en razón a que las ciencias no operan desde la evidencia, no puede ser un problema para la Biología, sino para la Filosofía" (Valencia, 1989).

Tal ha sido el trabajo alrededor de este interrogante, que hoy en día ya se han establecido algunas ( por no decir la mayoría ) características de lo vivo; de hecho si actualmente se consulta en un texto de Biología acerca de ¿qué es lo vivo? es muy probable encontrar algunas listas que resuman dichas características, entre las que muy seguramente se encontrará: “Los seres vivos se caracterizan por tener una organización química y estructural específica, por poder usar materiales de su ambiente para proveer energía y elementos estructurales para sus actividades celulares (metabolismo), por poder eliminar ciertos productos de su metabolismo (excreción), por su habilidad para producir réplicas de sí mismos (reproducción), por la facultad de responder a estímulos del ambiente (sensorialidad), por su habilidad para moverse de alguna manera y su habilidad para adaptarse a una variedad de cambios en el entorno (Baker, 1980)

Esta es una respuesta al parecer completa y convincente, fruto del trabajo de tantos años y de diversos personajes que la han construido, respuesta que parece explicar de alguna manera qué es lo vivo; sin embargo, abordada de este modo, en el contexto de este escrito, es una respuesta incompleta, pues carece de una perspectiva histórica que de cuenta del desarrollo de la misma y de su magnitud.

Este tipo de respuesta es muy común actualmente cuando se trata de abordar algún concepto. "En cualquier informe de la ciencia contemporánea, ya se trate de Biología 
Molecular o de Neurobiología, por citar algunos ejemplos, por obligación se toca ligeramente la superficie de incontables supuestos implícitos" (Smith, 1975), es decir que, en el afán de seguir avanzando en la producción de nuevos conocimientos científicos, generalmente se deja de lado o se trata superficialmente el desarrollo histórico del conocimiento construido hasta el momento; situación que en cierta medida repercute en otros contextos que toman como base estos estudios para su desarrollo. Por ejemplo cuando se quiere tener un acercamiento a un concepto desde otro contexto como el escolar, tampoco se tiene en cuenta el desarrollo histórico del mismo.

Podría afirmarse que la ausencia de una perspectiva histórica de los conocimientos que se enseñan constituye un elemento del común denominador en la escuela. "Actualmente diversas investigaciones muestran cómo el conocimiento científico es deformado por algunas prácticas pedagógicas que remplazan el conocimiento por lecciones, haciendo de aquel un saber fundamentalmente informativo que promueve la demanda de lo inmediato" (Valencia, 1989), de lo ya construido, sin darle la oportunidad al estudiante de conocer el proceso de dicha construcción. El abordaje de lo vivo en la escuela no escapa a esta situación, “generalmente el estudio de los seres vivos 0en nuestro sistema escolar es abordado desde una fase terminal de la indagación sobre lo vivo, sin entender que este concepto fundamental para el saber biológico requiere de un proceso de construcción del fenómeno viviente a través de la historia" (Chaparro, Garzón y Valencia, 1998).

En este orden de ideas, desde el problema del interrogante de lo vivo, su tratamiento ahistórico en las ciencias y en la escuela sobre todo; y considerando que "el estudio de la manera como se han construido y como se siguen construyendo los conocimientos científicos puede aportar pistas fundamentales para por un lado, transformar estos conocimientos en contenidos del proceso enseñanza-aprendizaje, y por otra parte idear las mejores rutas para desarrollar este proceso" (Chávez, 2002), a continuación se presenta una descripción del desarrollo histórico de las explicaciones más conocidas al interrogante de lo vivo y algunas implicaciones didácticas de abordarlo ahistóricamente e históricamente en la escuela.

\section{DESARROLLO HISTÓRICO DEL CONCEPTO DE LO VIVO.}

Algunas personas presentan una idea de la historia como aquella disciplina que relata una secuencia de hechos ocurridos en tiempos pasados, idea en la que se hace manifiesta la historia como una película donde los hechos se siguen los unos a los otros de una manera lineal, continua e incluso sumativa. No obstante, si se hace una reflexión sobre la dinámica desarrollada en los hechos históricos es evidente que la historia no es un proceso lineal continuo y aditivo. "Los hechos históricos no se suceden los unos a los otros de una forma simple en que un conocimiento nuevo reemplaza al anterior, sino que estos conocimientos se interconectan y relacionan a manera de una red compleja de causas y efectos" (Chávez, 2002).

Según Canguilhem (1993, Citado por: Chávez, 2002), la historia de las ciencias se constituye en una teoría explicativa de la evolución de los hechos. Se fundamenta en el análisis crítico y profundo de éstos, poniendo en relieve los saltos, los estancos, los errores, las rupturas y las 
regresiones de los postulados propuestos en cada momento. Dinámicas propias de las ciencias como resultado de un proceso discontinuo.

Estudiar las ciencias, en especial la Biología, desde esta perspectiva permite comprender que “éstas no son el resultado de una acumulación de saber en donde los logros de cada época son el escalón para nuevas formulaciones, sino el ejercicio de una racionalidad que encuentra su expresión en la confrontación de posiciones, conceptos y teorías con el fin de producir un conocimiento" (Valencia, 1989). De esta manera se puede decir, que el conocimiento de la Biología, y por tanto lo que hasta el momento se ha construido acerca del concepto de lo vivo como objeto de conocimiento de esta ciencia, se ha constituido como tal porque en el transcurso de la historia ha sido resultado de un proceso discontinuo.

\section{El estudio de la vida y el animismo.}

Antes del siglo XIX lo vivo no existía como objeto de conocimiento ni tampoco la Biología como ciencia (Valencia, 1989), sin embargo, al estar los seres humanos en contacto con la naturaleza tratando con animales, plantas, y otros organismos vivos, desde muchos siglos atrás ya se estaban preguntando acerca de ¿qué era la vida? incluso ya estaban trabajando en una respuesta a este interrogante. Trabajos que en cierta medida al ir generando cambios en la manera de mirar el fenómeno viviente, de cuestionarlo, fueron el primer escalón en el proceso que ha llevado a la constitución de la Biología como ciencia, su desarrollo y por tanto de lo vivo como su objeto de conocimiento (Valencia, 1989).

En un principio no existía una distinción clara entre la vida de un organismo y el espíritu de un objeto natural no vivo. "Casi todos los pueblos primitivos creían que existían espíritus en las montañas, en los ríos, en los animales y en las personas" (Mayr, 1995). Con el tiempo, este concepto de la naturaleza fue desapareciendo, los animales junto con las montañas, los ríos y los árboles ya no poseían espíritus. Sin embargo, en los tiempos de Aristóteles hasta el siglo XVI se siguió creyendo que en los seres vivos existía algo que los distinguía de la materia inanimada y que se separaba del cuerpo en el momento de la muerte, en este sentido el fenómeno viviente se explicaba desde el animismo, es decir, "se consideraba a los seres vivos como animados, dotados de un alma" (Castro, 2005), se pensaba lo animado en una relación de continuidad, de prolongación con lo inanimado y se presentaba el mundo como un todo jerarquizado (Jacob, 1999).

En esta época se pensaba que los seres no se reproducían, eran engendrados, la generación era siempre el resultado de una creación divina, atribuyéndole la formación de todos los individuos de una misma especie a una serie de creaciones simultáneas, realizadas sobre un mismo modelo en la creación del mundo (Jacob, 1999).

El conocimiento del mundo vivo apenas cambió de la Antigüedad al Renacimiento, los estudiosos de esta época en pocas palabras repetían lo que ya decía Aristóteles. En el siglo XVI los seres vivos se describían como una combinación de materia y forma, el orden que se establecía en los seres vivos no se distinguía del que reinaba en el universo, la diferencia 
evidente que distinguía los seres vivos de los minerales, era aquella de la que sólo el alma podía dar cuenta. Para esta época era difícil definir donde empezaba un grupo y donde acaba el otro, era difícil establecer si una esponja era una planta o un animal, las partes de las plantas y los animales eran análogas (las raíces se asemejaban al a boca, las hojas a los pelos) razón por la cual aún no existía la especie en el sentido en que la entendería la época neoclásica. Para explicar la formación de un ser era necesario recurrir a la acción de Dios. Para el siglo XVI la generación espontánea era comprensible, donde la implantación del alma en la materia del cuerpo daba pie a la generación (Jacob, 1999).

\section{El mecanicismo y los organismos como máquinas.}

“¿Qué es la vida? y ¿como se pueden explicar los procesos vitales? han sido temas controversiales desde el siglo XVI. En pocas palabras la situación a mediados de este siglo y en el siglo XVII estaba representada por el enfrentamiento entre dos formas de darle respuesta a este interrogante. Por un lado existía un bando que afirmaba que, en realidad, los organismos no eran diferentes de la materia inanimada por lo cual podían ser estudiados desde la física y la química; a estas personas se les llamó mecanicistas. Por otra parte un bando contrario, los llamados vitalistas, aseguraba que los organismos tenían propiedades que no existían en la materia inerte, y que por lo tanto la explicación de los procesos vitales no se podía reducir a las leyes de la física y la química. En algunos periodos los mecanicistas parecieron salir victoriosos mientras que en otros tiempos fueron los vitalistas, no obstante actualmente es claro que ambos bandos tenían en parte la razón así como también en parte se equivocaban" (Mayr, 1995).

En el siglo XVII ya no era posible situar en un mismo plano la forma de una planta o un animal, el interés no se centraba ya en la creación divina de la naturaleza sino en su funcionamiento, en el descubrimiento de su orden. Descartes planteaba que para poder encontrar dicho orden era necesario reducir la naturaleza a la simplicidad de las unidades que la componían y en este sentido poder descifrar las regularidades de sus fenómenos y establecer las leyes que regían su funcionamiento, las cuales podían ser representadas a partir de un lenguaje matemático (Jacob, 1999). Esta nueva manera de contemplar el mundo desde los trabajos de Galileo, Descartes, Newton entre otros, en la que los astros, las piedras, los animales, las plantas obedecían a las leyes de la mecánica expresadas por el cálculo, constituyó una visión mecanicista de los fenómenos vivientes, lo cual culminó con la afirmación cartesiana de que todos los organismos no eran más que máquinas (Mayr, 1995).

En este siglo surgieron la fisiología, derivada de la medicina y la historia natural, como las corrientes guía en el estudio de los seres vivos desde su estructura visible (Jacob, 1999); corrientes libres de superstición y magia que elaboraron un lenguaje fundamentalmente taxonómico de los seres, el cual fue trabajado hasta comienzos del siglo XIX, época en la que con la transformación de este lenguaje, el desarrollo de las teorías celular, evolutiva y genética respectivamente, se abrió la posibilidad de acceder a los seres vivos mas allá de lo visible, desde relacionar lo que se ve con lo que no se ve, desde su organización (Valencia, 1989). 


\section{Más allá de lo visible: El vitalismo y el organicismo}

Bajo este panorama surge el vitalismo, como aquella corriente basada en el concepto de organización que intentó plantear de nuevo la naturaleza de la vida, que intentó presentar argumentos científicos contra la teoría maquinista de los organismos (Mayr, 1995). El vitalismo se opuso a la reducción de los seres vivos a la física y a la química, manteniendo que si bien estas leyes se pueden aplicar a los organismos resultan insuficientes para la plena comprensión del fenómeno de la vida y que la organización de un organismo como un todo no puede ser comprendida únicamente desde el estudio simplista de sus partes, asegurando así que existe alguna entidad (protoplasma o entelequia) no física o alguna fuerza o campo que debe sumarse a las leyes fisicoquímicas para la comprensión de la vida (Capra, 1998).

Con el tiempo, el vitalismo fue adquiriendo fuerza, a medida que se desarrollaron sus postulados en contra del mecanicismo y se plantearon teorías como la Flogística de la combustión de Ernst Stahl, y la de la epigénesis de Friedrich (la cual invocaba un agente causal que trasformaba el huevo fecundado en un adulto) que en parte aportaron respuestas razonables al interrogante de la vida; sin embargo, al parecer el vitalismo un concepto metafísico que no ofrecía pruebas científicas y al desarrollarse nuevos conceptos biológicos, como la genética y la selección natural, que explicaban los fenómenos que solían citarse como pruebas del vitalismo éste cayó en un rápido declive (Mayr, 1995).

Así, desde finales del siglo XVIII y principios del XIX aparece la Biología como aquella ciencia de la cual lo vivo se constituye objeto de conocimiento, esto como resultado de ver en el fenómeno viviente algo que se escapaba a una simple explicación física y química, algo que trascendía lo visible, algo más que una máquina (Castro, 2005).

Es así, que la caída del vitalismo lejos de significar la victoria del mecanicismo dio lugar a un nuevo sistema explicativo de lo vivo, basado también en la organización, el organicismo. Esta nueva corriente aceptaba que los procesos vitales a nivel molecular se podían explicar perfectamente por mecanismos fisicoquímicos, pero que dichos mecanismos tenían una influencia cada vez menor, sino nula en los niveles superiores de integración; es decir las características exclusivas de los organismos no se debían a su composición sino a su organización (Mayr, 1995) la cual hace referencia a las relaciones internas que se dan entre las partes constituyentes de los mismos (Jacob, 1999); planteamiento que según Needham (Citado por: Capra, 1998) condujo a la búsqueda de relaciones organizadoras a todos los niveles de la estructura viviente, considerando que una de las características clave de la organización de los organismos era su naturaleza jerárquica, lo cual significa la tendencia a construir estructuras multinivel, niveles de organización.

En este sentido el organicismo demostró que los seres vivos no se podían estudiar desde sus partes, pues al hacerlo de esta manera se pierde la organización, se pierden las relaciones que surgen de la interrelación de éstas como consecuencia de sus propiedades. Es la organización de las partes la que controla el todo, por eso la base del organicismo era el hecho de que los seres vivos poseen organización, que no son simples montones de caracteres o de moléculas, 
porque su funcionamiento depende por completo de sus interrelaciones mutuas, de sus interacciones e interdependencias, en fin de su organización (Mayr, 1995).

En este orden de ideas, "al sustituir la estructura visible por la organización como objeto de análisis, se introduce en el estudio de los seres vivos un sistema de referencia para los datos inmediatos de la percepción. Si para el siglo XIX la vida se identifica con la organización es porque ésta constituye un centro de articulación entre la estructura, la función y el medio, donde la vida sólo existe en la medida en que estos tres parámetros están en armonía y si alguno varía influye en el conjunto del organismo". (Jacob, 1999)

\section{Una nueva manera de pensar lo vivo: El pensamiento sistémico y la Biología molecular.}

Las ideas propuestas por los organicistas contribuyeron al nacimiento de una nueva manera de pensar, el pensamiento sistémico, corriente que comparte los postulados del organicismo y que adicionalmente plantea que los organismos son sistemas vivos en términos de conectividad, relaciones y contexto. Según esta visión las propiedades esenciales de un organismo o sistema viviente son propiedades del todo que ninguna de las partes posee. Emergen de las interacciones y relaciones entre las partes. Estas propiedades son destruidas cuando el sistema se disecciona, razón por la cual los sistemas no pueden ser comprendidos por medio del análisis. En el pensamiento sistémico las propiedades de las partes sólo se pueden comprender desde la emergencia, desde la organización del conjunto (Capra, 1998).

Por otro lado, en la segunda mitad del siglo XX, el desarrollo de la Biología Molecular ha permitido explicar lo vivo a partir de la estructura y función de sus macromoléculas, a esta nueva concepción se le ha denominado neomecanicismo dado su reduccionismo molecular. Esta corriente plantea que los organismos son máquinas, sólo que a diferencia del mecanicismo del siglo XVII se construyen a sí mismas. En este sentido, el fenómeno vivo a partir de la Biología Molecular se presenta desde los componentes moleculares de los organismos y de las reacciones que en ellos tienen lugar (Castro, 2005).

Aunque esta visión parezca retroceder en el tiempo, no es lo que parece literalmente, actualmente la Biología molecular aunque retoma algunos aspectos del mecanicismo, está acercándose a la elaboración de una nueva hipótesis de trabajo que reinterpreta los procesos moleculares de la vida a la luz de la teoría de los sistemas autónomos abiertos organizados jerárquicamente, estableciéndose una complementariedad que va mas allá de las corrientes anteriores y reconcilia el aparente reduccionismo de la Biología molecular con algunos aspectos holísticos desarrollados por la teoría general de los sistemas (Andrade, 1994).

En este sentido en la actualidad, cuando se consulta a biólogos o a filósofos de la ciencia parece existir un consenso sobre los organismos. A nivel molecular, todas sus funciones y a nivel celular, casi todas obedecen las leyes de la física y la química, no hay ya ninguna explicación que retome la existencia de una fuerza vital para explicar lo vivo, así como los organismos no son reducibles a máquinas, los organismos son fundamentalmente diferentes de la materia inerte, son sistemas ordenados jerárquicamente con numerosas propiedades 
emergentes, sus actividades están gobernadas por programas genéticos que contienen información adquirida a lo largo del tiempo y además poseen algunos fenómenos específicos tales como: programas evolucionados, mecanismos reguladores, sistemas teleonómicos, orden de magnitud limitado, ciclo vital y sistemas abiertos (Mayr, 1995).

La búsqueda de respuestas al interrogante de lo vivo ha sido uno de los trabajos mas duraderos y controversiales a lo largo de la historia de la Biología. Numerosas respuestas fueron elaboradas y mientras algunas de ellas fueron complementarias otras lo fueron contradictorias. Algunas fueron olvidadas, otras retomadas, otras aún están presentes y otras aún están por elaborarse; pero así se construye el conocimiento científico, afortunadamente así es la historia de las ciencias, hay muchos conceptos en Biología y en otras ciencias de los cuales se puede representar un desarrollo histórico no muy diferente al concepto de lo vivo. Siendo en este desarrollo histórico en donde se encuentra la verdadera riqueza del concepto, lo que realmente debe ser enseñado.

\section{Algunas implicaciones didácticas del tratamiento ahistórico del concepto de lo vivo}

Es curioso ver como se mencionaba anteriormente la manera en que "el conocimiento científico es deformado por ciertas prácticas pedagógicas que reemplazan los descubrimientos por lecciones, haciendo de aquel un saber fundamentalmente informativo, que promueve la demanda de lo inmediato y lo económicamente rentable, y que en consecuencia, inserta al estudiante en una sociedad competitiva... y que le niega la oportunidad de preguntarse por el sentido de la disciplina que estudia" (Valencia, 1989).

Comúnmente en la escuela se enseñan los conceptos tal cual han sido planteados, sin tener en cuenta su desarrollo histórico, se les enseña a los estudiantes el conocimiento ya elaborado, incluso atribuyéndoselo a un solo personaje, que además al parecer lo descubrió por casualidad, dejando de lado el desarrollo de los conceptos a través del tiempo, los cambios, las controversias, las discusiones, los campos de validez, las dificultades de su construcción, entre otros aspectos que son comunes a la dinámica de la elaboración de conocimiento científico, como se acaba de evidenciar con el concepto de lo vivo (Giordan et al, 1988).

La investigación en enseñanza de las ciencias ha sentado como precedente la vinculación de la historia como un elemento importante para la enseñanza de las mismas. Esta vinculación tendría como propósito aproximar a los estudiantes a las problemáticas que se han suscitado a lo largo de la práctica científica para la comprensión de la producción de conceptos científicos (Muñoz, 2005) y así dotar de rigurosidad el desarrollo de dicho conocimiento, e incluso brindar la posibilidad de entender mejor un concepto, puesto que muchas veces resulta más significativo conocer cómo se ha llegado al concepto como tal, que simplemente aprenderse de memoria el dato de qué significa y qué personaje lo "descubrió".

El reconocimiento y comprensión de lo vivo es fundamental en los primeros años escolares, pues es en este momento cuando los estudiantes comienzan a preguntarse cosas acerca de su 
cotidianidad, inquietudes que según Castaño y Leudo (1998) se ha demostrado son inicialmente acerca de los fenómenos naturales y especialmente sobre lo vivo.

No obstante, en la escuela al centrarse la didáctica en la transmisión de contenidos ahistóricos y el aprendizaje en el rendimiento, los intereses espontáneos del estudiante que giran alrededor de lo vivo y de los fenómenos de la naturaleza no se tienen en cuenta, ni tampoco se centra la atención en las formas como éste construye pensamiento acerca de este concepto (Castaño y Leudo, 1998).

Generalmente el estudio de los seres vivos en la escuela es abordado desde una fase terminal de la indagación sobre lo vivo, por ejemplo la célula. Sin entender que este concepto fundamental para el saber biológico requiere de un proceso de construcción del fenómeno viviente, en donde maestros y estudiantes se involucren en la tarea de reconceptualizar la historia, asumiendo un particular punto de vista que permita consolidar una visión, en la cual, aspectos que generalmente no se tienen en cuenta como la historia de las ciencias se planteen como prioritarios y dinamizadores del conocimiento (Chaparro, Garzón y Valencia, 1998).

Algunas investigaciones (Castaño y Leudo, 1998) han demostrado que la mayoría de los estudiantes, entre los cinco y ocho años de edad, tienen una visión propia acerca de lo vivo, aunque obviamente diferente al actual concepto biológico académicamente constituido. Este pensamiento se manifiesta en que para definir lo vivo los niños acuden a lo evidente que inicialmente es el movimiento y en que paulatinamente su pensamiento va dirigiendo la atención a otras características igualmente evidentes e inmediatas, como el crecimiento, la necesidad de alimentarse, el tener hijos o el morir.

Es de esperarse que en la escuela los estudiantes se acerquen a la comprensión de lo vivo a partir del concepto biológico académicamente constituido; sin embargo, es evidente que esta escuela moderna en su afán de enseñar más contenidos y así mejorar el rendimiento académico de los estudiantes le muestra al niño un concepto acabado, un concepto sin historia, sin controversias, sin esfuerzos, sin interrogantes (Castaño y Leudo, 1998), un concepto que éste debe repetir, lo que no le posibilita poner en cuestión lo que el estudiante ha construido de lo vivo durante su experiencia, y por tanto le niega una verdadera comprensión de la nueva explicación que se le presenta, asumiéndola momentáneamente para repetir en un examen y dar cuenta de su rendimiento (Valencia, 1989).

A diferencia de lo que promueve la escuela, se ha demostrado que no es posible un conocimiento directo de los fenómenos. Conocer no es ni reproducir, ni representar; conocer es construir, pues toda referencia al mundo implica mínimamente un acto de clasificación y de ordenación (Melich, 1996, Citado por: Castaño y Leudo, 1998). Como plantea Kleiber (Citado por: Castaño y Leudo, 1998) es así como las estructuras conceptuales provienen de nuestra experiencia, de la categorización, operación, sin la cual el entorno percibido sería caótico y permanentemente nuevo. De ahí se sigue que la Biología no existiría como ciencia, 
de no ser por la categorización de la inmensa diversidad de lo vivo que se elaboró a través del tiempo (Castaño y Leudo, 1998).

Es así, como desde el problema del interrogante de lo vivo, su tratamiento ahistórico en las ciencias, en la escuela y las implicaciones didácticas que esto conlleva, se hace más que evidente la necesidad de generar en la escuela, alternativas pedagógicas que posibiliten la enseñanza del desarrollo histórico epistemológico de los conceptos. Alternativas que posibiliten al estudiante desarrollar un pensamiento propio, debatir, interrogarse, utilizar su imaginación e intentar la búsqueda de soluciones a sus propias inquietudes, en fin como dirían Castaño y Leudo (1998) alternativas que permitan desarrollar en el estudiante un pensamiento distinto al que la escuela pretende imponer.

Afortunadamente el desarrollo de la didáctica de las ciencias, ha permitido evidenciar las posibles aportaciones de la historia y epistemología de las ciencias en la enseñanza de las ciencias. Con esto, no sólo haciendo referencia simplemente al reconocimiento del papel educativo que estas disciplinas pueden tener y que ha sido resaltado por científicos, historiadores de la ciencia y educadores, sino el papel que la historia y la epistemología pueden jugar en el desarrollo de un cuerpo específico de conocimientos didácticos; resaltando que las aportaciones en este campo han sido relativamente escasas hasta hace poco, pero el desarrollo de la didáctica de las ciencias ha terminado por impulsar una línea de investigación centrada en las relaciones entre historia y epistemología de las ciencias y enseñanza de las ciencias. Es así como, las renovaciones de la enseñanza de las ciencias debe a la historia y epistemología de las ciencias la idea de una creciente aproximación de las situaciones de aprendizaje a una actividad científica, permitiendo que los estudiantes disfruten con la ciencia, adquieran una visión mas clara de lo que hacen los científicos, e incluso sean impulsados a seguir estudios científicos de nivel superior (Gil Pérez, 1993).

El tratamiento ahistórico de las ciencias dejará de ser un común denominador de la escuela cuando los profesores dejen de permitirlo mediante las prácticas pedagógicas que desarrollen en el aula, prácticas que son esbozadas en parte desde el conocimiento profesional del profesor construido durante la formación inicial; en este sentido y no solo haciendo referencia a la historia y epistemología de las ciencias, si no incluyendo también otros aspectos propios de la Biología que deben tenerse en cuenta para guiar los contenidos y los procesos de su enseñanza es de resaltar la importancia de incluir estos aspectos en los programas de formación inicial de profesores de Biología, aspectos que aportaran elementos importantes para salvar algunas falencias de la escuela competitiva, "ofreciendo posibilidades para hacer de los estudiantes personas diferentes, críticas, cuestionadotas, polémicas, y en todo caso hombres, no esclavos" (Valencia, 1989).

\section{Bibliografía.}

- ANDRADE, L. 1994. Holismo y reduccionismo en el desarrollo de la biología molecular. Memorias. Seminario Nacional "El quehacer teórico y las perspectivas holistas y reduccionistas". Academia colombiana de Ciencias Exactas, Físicas y naturales. Bogotá. 
- BAKER, J.J.W.; G.E. Allen. 1980. Biología e investigación científica. Fondo Educativo Interamericano, S.A. México. 666 p.

- CAPRA, F. 1998. La trama de la vida. Una nueva perspectiva de los sistemas vivos. Anagrama. Barcelona. 359 p.

- CASTAÑO, N.; LEUDO, M. 1998. Las nociones de los niños acerca de lo vivo. Implicaciones didácticas. Revista Ciencia y Tecnología. № 4.

- CASTRO, J. 2005. La biología molecular una nueva concepción de lo vivo. Revista Ciencia y Tecnología. № extra.

- CHAPARRO, C.; GARZÓN, J., VALENCIA, S. 1998. Lo visible y lo oculto en torno al pensamiento sobre lo vivo. Revista Física y Cultura: Cuadernos sobre historia y enseñanza de las ciencias. № 4 .

- CHÁvEZ, M. 2002. Estudio analítico no lineal de los modelos explicativos de la nutrición vegetal y su valor para el proceso de enseñanza-aprendizaje. Revista Ciencia y Tecnología. № 11 .

- GIL PÉREZ, D. 1993. Contribución de la historia y de la filosofía de las ciencias al desarrollo de un modelo de enseñanza/aprendizaje como investigación. Revista Enseñanza de las Ciencias. Vol. 11. № 2.

- GIORDAN, A.; RAICHVARG, D.; DROUIN, J.; GAGLIARDI, R.; CANAY, A. 1988. Conceptos de Biología. 1. La respiración. Los microbios. El ecosistema. La neurona. Labor. Barcelona.

- JACOB, F. 1999. La lógica de lo viviente. Una historia de la herencia. Tusquets editores. España. 320 p.

- MAYR, E. 1995. Así es la Biología. Debate S.A. España. 328 p.

- MUÑOZ, D. 2005. El tratamiento didáctico de la historia de las ciencias en los libros de texto. Revista Ciencia y Tecnología. № Extra.

- SCHRODINGER, E (1984) ¿Qué es la vida? Barcelona: Tusquets.

- SMITH, C. 1975. El problema de la vida. Ensayo sobre los orígenes del pensamiento Biológico. Alianza Editorial. Madrid. 448 p.

- VALENCIA, S. 1989. La Biología; ¿ciencia de la vida o ciencia de lo vivo? Análisis de condiciones epistemológicas que hicieron posible pensar los determinantes del fenómeno vivo como el objeto de la Biología. Trabajo de grado de Licenciatura en Biología. Universidad Pedagógica Nacional, Facultad de Ciencia y Tecnología, Bogotá.

- VARELA, F. 2002. El fenómeno de la vida. Dolmen. 2da edición. España. 